\title{
A Complete Geometric Representation of Four-Player Weighted Voting Systems
}

\author{
Zhengjia Jiang $^{1}$ \\ ${ }^{1}$ Fishkill, New York, United States \\ Correspondence: Zhengjia Jiang, Fishkill, New York, United States. E-mail: jiangzhengjia@ gmail.com
}

Received: January 4, 2013 Accepted: February 3, 2013 Online Published: February 21, 2013

doi:10.5539/jmr.v5n1p122 URL: http://dx.doi.org/10.5539/jmr.v5n1p122

\begin{abstract}
This paper seeks to expand voting power theory, a branch of game theory that applies to many important organizations. Typically, weighted voting systems are displayed using the algebraic representation, consisting of a quota and a weight vector. A newer idea, however, is the geometric representation. This representation maps all normalized weighted voting systems onto a simplex and thus can be called a complete representation of weighted voting systems. The concept of the region, sets of characteristically identical weighted voting systems, will be introduced, greatly simplifying the analysis of weighted voting systems. In this paper, four-player weighted voting systems are solved completely using the geometric representation. The geometric representation will be shown to be a useful alternative to the algebraic representation.
\end{abstract}

Keywords: weighted voting, voting systems, geometry, four-player games

\section{Introduction}

Weighted voting systems form the basis of many organizations, such as corporations, the International Monetary Fund, and the United States Electoral College. A branch of game theory, known as voting power theory, includes the analysis of weighted voting systems. Most typically, weighted voting systems are represented algebraically by a quota and a weight vector, showing the weights of the players and the total weight needed to pass a measure. The purpose of weighted voting is to give different players different amounts of influence over decisions. Though players with more weight will never have less power than players with less weight, other details of the power distributions for weighted voting systems have unclear relationships with the quotas and weight vectors. Thus, various power indices have been invented to measure voting power. Interestingly, very few indices calculate the power distribution directly from the algebraic representation: usually, the algebraic representation is first converted into some other representation.

The algebraic representation can be used to analyze weighted voting systems in various other ways. Laruelle and Valenciano (2005) elucidated paradoxes of voting power through algebraic manipulation. They explain counterintuitive results by exposing the algebra that leads to them. Tolle (2003) produced a full analysis of four-player weighted voting systems, though he only considered schemes where no player holds veto power. In his paper, he poses several questions. Cuttler et al. (2005), Elkind et al. (2009), and Keijzer et al. (2010) all attempted to find an algorithm that generates all feasible weighted voting systems for a certain number of players, a discovery that would answer Tolle's "inverse problem": given a desired power distribution, can a weighted voting system resulting in the desired power distribution be created? The algebraic representation of weighted voting systems is concise and easy to display, so it is useful in analysis.

All reasonable power indices, however, will reveal that an infinite number of weighted voting systems with different algebraic representations will result in the same power distribution. Besides explaining why most power indices cannot calculate voting power directly from the quota and weight vector, this fact also reveals that many weighted voting systems are equivalent because they are characterized by the same set of winning coalitions. One may also recognize that even more basic than the set of winning coalitions is the set of minimum winning coalitions. Papers such as Kirsch and Langner (2009) and Bishnu and Roy (2009) sought to find ways to directly calculate the power distribution or hierarchy of players in a weighted voting system using only the set of minimum winning coalitions, a representation of weighted voting systems that is more revealing than the algebraic representation.

Other papers sought to use a geometric representation. Different papers such as Kirstein (2009) and Brown et al. 
(2009) used geometric analysis to supplement their algebraic manipulations. The former focused on three-player scenarios to apply voting power theory to a dispute. The latter was a response to Tolle (2003), seeking to find an upper bound on the number of feasible power distributions in $n$-player weighted voting systems. Though primarily employing algebraic techniques, the two papers also included geometric representations of weighted voting systems.

One paper which employed a geometric representation of weighted voting systems as its primary tool was Jones (2009). Exploring the same topic as Laruelle and Valenciano (2005), Jones (2009) employed geometric analysis to shed light on paradoxes of voting power from a new angle. Though this paper worked primarily with three-player examples, his representation of weighted voting systems was very versatile: it can be generalized for any number of players and its non-exploitation of symmetry makes it a complete portrayal of $n$-player weighted voting systems.

In Section 2, this paper will discuss preliminary information, such as definitions and notation. In Section 3, the Geometric Representation is introduced formally. In Section 4, the concept of the "Region" is explored. In Section 5 , a brief overview of three player weighted voting systems is given, followed by a brief overview of four player weighted voting systems in Section 6. The Conclusion is Section 7. More details about four player weighted voting systems are found in the appendixes.

\section{Preliminaries}

\subsection{Definitions}

The voting games in this paper are games in which a set of players, $\mathcal{S}=\{1,2, \ldots, n\}$, vote on a binary decision. Those in favor of the decision will form a coalition $C \in \mathrm{P}(\mathcal{S})$, where $\mathrm{P}(\mathcal{S})$ denotes the power set of $\mathcal{S}$. Certain coalitions will win, while others will lose. The set of winning coalitions is denoted $\mathcal{W}$. If $C \in \mathcal{W}$, then $C$ is a winning coalition. Otherwise, $C$ is losing. A voting game is then a pair $(\mathcal{S}, \mathcal{W})$.

Weighted voting systems can be simple, proper, and/or weighted. A voting game is simple if the following conditions hold:

(1) $\emptyset \notin \mathcal{W}$;

(2) $\mathcal{S} \in \mathcal{W}$

(3) If $\mathcal{A} \subseteq \mathcal{B}$ and $\mathcal{A} \in \mathcal{W}$, then $\mathcal{B} \in \mathcal{W}$.

In words, a simple game satisfies the following conditions: the null coalition of no players is losing, the grand coalition of all players is winning, and any superset of a winning coalition is winning (satisfying monotonicity).

A voting game is proper if:

(1) $\mathcal{A} \in \mathcal{W} \rightarrow \mathcal{S} \backslash \mathcal{A} \notin \mathcal{W}$.

A simple proper voting game satisfies the condition that no two disjoint coalitions can be winning at once, a property important for practical applications. Notice that by monotonicity, a simple voting game's set of minimum winning coalitions must form an antichain. However, not all antichains will result in sets of minimum winning coalitions that characterize simple proper voting games.

A voting game is weighted if it can be written in the form $(q, \mathbf{W})$, where $\mathbf{W}=\left[w_{1}, w_{2}, \ldots, w_{n}\right]$ and the following conditions are satisfied:

(1) $q>0$;

(2) $w_{i} \geq 0$ for all $i \in\{1,2, \ldots, n\}$;

(3) $\sum_{i \in C} w_{i} \geq q, \forall C \in \mathcal{W}$;

(4) $\sum_{i \in C} w_{i}<q, \forall C \notin W$.

The weight of a coalition is equal to the sum of the weights of its members. A coalition is winning if and only if its weight is not less than the quota. All games that are simple and proper that have five or less players are weighted. Since this paper focuses on games with four or less players, all games in this paper are simple, proper, weighted voting systems.

As stated in the introduction, most often, a weighted voting system is represented algebraically as the pair $(q, \mathbf{W})$, but it is typically notated as $\left[q ; w_{1}, w_{2}, \ldots, w_{n}\right]$. 


\subsection{The Banzhaf Power Index}

Much of voting power theory is devoted to the measuring of voting power; the power of player $i, p_{i} \in[0,1]$, reflects the influence player $i$ has over the outcome. A player with a voting power of 0 is a dummy with no influence over the outcome. A player with a voting power of 1 is a dictator whose sole decision determines the outcome.

Each voting power index, however, defines voting power using different parameters, and so these indices will almost always disagree on the ratio of power between pairs of players and sometimes even the power hierarchy of players. This paper will use the Banzhaf power index, introduced in Banzhaf (1965).

The Banzhaf power index equates power with swing votes - if a coalition $C$ containing player $i$ is winning, yet coalition $C /\{i\}$ is losing, then player $i$ is said to have a swing vote. To mathematically define the Banzhaf power of a player, we introduce a function $\chi(C): \mathrm{P}(\mathcal{S}) \rightarrow\{0,1\}$. The function $\chi$ satisfies the following properties:

(1) $\chi(C)=1, \forall C \in W$

(2) $\chi(C)=0, \forall C \notin W$.

The swing count for player $i, \beta_{i}$, is defined as follows:

$$
\beta_{i}=\sum_{C: i \in C}[\chi(C)-\chi(C \backslash\{i\})] .
$$

And the Banzhaf power, $p_{i}$, is defined as follows:

$$
p_{i}=\frac{\beta_{i}}{\sum_{j=1}^{n} \beta_{j}} .
$$

The Banzhaf power index measures a player's voting power by measuring that player's proportion of swing votes.

\subsection{Notation}

When this paper discusses an $n$-player weighted voting system, it will be represented algebraically in the form $\left[q ; w_{1}, w_{2}, \ldots, w_{n}\right]$. The set of players will be $\mathcal{S}=\{1,2, \ldots, n\}$. In general, all of the conventions used in section 2.1 will be used as well.

When this paper discusses 3-player weighted voting systems, however, certain simplifications will be made. The players will be known as $A, B$, and $C$. When the letters $A, B$, and $C$ appear in equations or inequalities, they will denote the weight of the corresponding player. A coalition will be notated by enclosing its members in brackets, e.g. $[A C]$ represents the coalition containing players $A$ and $C$. A coalition appearing without brackets indicates the weight of that coalition, e.g. $A C$ denotes the weight of coalition $[A C]$.

In 4-player weighted voting systems, the same notation as for 3-player weighted voting systems will be followed, except the players will be $A, B, C$, and $D$.

\section{The Geometric Representation}

\subsection{A Need for Normalization}

The only characteristic of a weighted voting system significant in finding its Banzhaf power distribution is its set of winning coalitions, or more basically, its set of minimum winning coalitions. Since this set determines the distribution of swing votes, any modification to a weighted voting system that maintains the set of minimum winning coalitions will not change the power distribution - in fact, the two games will function identically.

An infinite number of algebraic representations of weighted voting systems will result in the same minimum winning coalitions, so all of those games are equivalent. This observation suggests that only a finite number of possible "different" weighted voting systems exist for a finite number of players - a true statement. Several papers such as Brown et al. (2009) have tried to find an upper bound for this number for small, finite values of $n$.

Theorem 1 There are only finitely many "different" weighed voting systems for $n$ players.

Proof Sketch. A voting game is defined by its set of minimum winning coalitions. Since the number of players is finite, the number of possible coalitions, $2^{n}$, is also finite. The number of sets of coalitions is also finite, $2^{2^{n}}$, which is a (very generous) upper bound on the number of possible sets of minimum winning coalitions. Since each distinct weighted voting system corresponds to one set of minimum winning coalitions, the number of distinct weighted voting systems with $n$-players is also finite for any finite $n$. 
To decrease the number of possible algebraic representations while maintaining full flexibility, it becomes necessary to normalize the weighted voting system. Since the weighted voting systems $\left[q ; w_{1}, w_{2}, \ldots, w_{n}\right]$ and $\left[a q ; a w_{1}\right.$, $\left.a w_{2}, \ldots, a w_{n}\right]$ are functionally identical for any positive $a$, it makes sense to make no distinction between these weighted voting systems and select one game to represent all such games. The normalization will scale the weights and quota so that the sum of the weights is 1 and the quota is in the interval $\left(\frac{1}{2}, 1\right]$.

\subsection{Creating the Geometric Representation}

From the normalized algebraic representation, it is possible to convert a weighted voting system into its geometric representation. The geometric representation of a weighted voting system with $n$ players and quota $q \in\left(\frac{1}{2}, 1\right]$ consists of an $(n-1)$-dimensional simplex with unit height. The simplex represents all possible weight vectors. There will be a bijective mapping from all possible normalized weighted voting systems with $n$ players and quota $q$ to points contained on and in the $(n-1)$-simplex of the geometric representation for $n$-player weighted voting systems with quota $q$. Since the simplex shows all possible weighted voting systems for a certain value of the quota, the geometric representation can be called complete for each value of the quota.

Now consider the (n-1)-simplex with unit height. By definition, the (n-1)-simplex has $n$ facets, each of $(n-2)$ dimensions. These facets can be enumerated with the integers 1 through $n$, each facet representing one of the $n$ players. Each point represents the weighted voting system where $w_{i}$ (the normalized value) is equal to the length of the perpendicular segment from that point to the facet labeled with the integer $i$. If a point is on a particular facet $i$, then that point represents a weighted voting system where $w_{i}=0$. The sum of the lengths of the perpendicular segments from any point in the simplex with unit height to the facets is equal to 1 , the sum of all the weights, as proven in Theorem 2.

Theorem 2 The sum of the lengths of the perpendicular segments from any point on the $n$-simplex with unit height to its facets is equal to 1.

Proof. Let the hypervolume (the concept analogous to volume for higher dimensions) of the $n$-simplex be $V$. An $n$-simplex is essentially a regular $n$-dimensional pyramid. Then let the formula for calculating the hypervolume of the $n$-pyramid be expressed in the form $C B h$, where $C$ is some constant, $B$ is the hypervolume of the $(n-1)$ dimensional base, and $h$ is the height of the pyramid. Then let the hypervolume of each $(n-1)$-hyperface of the $n$-simplex be equal to $S$. Then the hypervolume of the $n$-simplex as calculated by treating the entire simplex as one pyramid is $V=C S(1)=C S$. However, the hypervolume of the $n$-simplex can also be calculated from an arbitrarily chosen point within the simplex. Draw lines extending from the arbitrarily chosen point to each vertex of the $n$-simplex, as well as the perpendicular segments (heights) from the chosen point to each facet. Then the $n$ simplex is divided into $n$ smaller pyramids, each with a different facet as its base and the chosen point as the vertex. Let the length of the perpendicular segments be equal to $a_{1}, a_{2}, \ldots, a_{n}$. Then the hypervolume of the $n$-simplex is calculated by summing the hypervolumes of each smaller pyramid:

$$
V=C S a_{1}+C S a_{2}+\cdots+C S a_{n}=\operatorname{CS}\left(a_{1}+a_{2}+\cdots+a_{n}\right) .
$$

Yet $V=C S$, so it follows that $a_{1}+a_{2}+\cdots+a_{n}=1$.

The geometric representation of a weighted voting system displays it as a single point in a continuous space. Some change in weights or the quota will not change a weighted voting system since the set of minimum winning coalitions will remain unchanged. Naturally, a question arises: how much can a weighted voting system be altered without changing the set of minimum winning coalitions? To answer this question, the concept of the region is introduced.

\section{Regions}

\subsection{Defining Regions}

The most convenient property of the geometric representation of weighted voting systems, besides its completeness, is the presence of the concept of the region. In the geometric representation for a weighted voting system with quota $q$, a region is defined to be a set of points in the simplex - or equivalently, a set of normalized weighted voting systems with quota $q$ - which return the same set of minimum winning coalitions, and thus are equivalent. A point is defined to be "in a region" if it returns the same set of minimum winning coalitions which characterize that region. The region provides an extremely visual method for the analysis of weighted voting systems.

Regions are bounded by the same conditions that define the sets of minimum winning coalitions that characterize them - the winning coalitions must all be winning and the losing coalitions must all be losing. As an example, 
consider the region in 3-player weighted voting systems characterized by the minimum winning coalitions set $[A B][A C]$. In this region, player $A$ can form a winning coalition with either one of the other two players. A weighted voting system must satisfy the following inequalities for it to belong to this region:

(1) $A B \geq q$;

(2) $A C \geq q$;

(3) $B C<q$;

(4) $A<q$.

The four inequalities above define half spaces. The intersection of these half spaces within the simplex is then the region. The inequalities that define each region explain much of the region's properties. Regions are limited, however, by how the quota needs to be fixed. To allow geometric analysis with a changeable quota, it is important to define the superregion. A superregion is defined as the set of all normalized weighted voting systems that yield the same set of minimum winning coalitions. The question of how to geometrically represent superregions will be explored later. Regions and superregions are like polytopes, but the half spaces whose intersection is the region or superregion can be either open or closed.

\subsection{Properties of Regions}

Regions and superregions have many properties advantageous for analysis. The theorem below summarizes many important properties of regions and superregions.

Theorem 3 If two weighted voting systems in the same superregion are $\left[a ; w_{a 1}, w_{a 2} \ldots\right]$ and $\left[b ; w_{b 1}, w_{b 2} \ldots\right]$, the following weighted voting system is also in the same superregion: $\left[a f+b(1-f) ; w_{a 1} f+w_{b 1}(1-f), w_{a 2} f+w_{b 2}(1-\right.$ $f)$...], where $0 \leq f \leq 1$.

Proof. Examine an arbitrary winning coalition. Without loss of generality, let it be the coalition consisting of players $1,2 \ldots$ and $n$ (this assumption can be fulfilled by permuting the players). By assumption, $w_{a 1}+w_{a 2}+\cdots+$ $w_{a n} \geq a$ and $w_{b 1}+w_{b 2}+\cdots+w_{b n} \geq b$.

By multiplying the first inequality by $f$ and the second by $(1-f)$, and then regrouping terms, the following is achieved:

$$
\left[w_{a 1} f+w_{b 1}(1-f)\right]+\left[w_{a 2} f+w_{b 2}(1-f)\right]+\ldots\left[w_{a n} f+w_{b n}(1-f)\right] \geq a f+b(1-f)
$$

This result demonstrates that any coalition which is originally winning will remain winning. Similarly, it is possible to prove that any losing coalition will remain losing by replacing the " $\geq$ " signs with " $<$ " signs. Hence, the theorem is true.

In other words, this theorem states that it is possible to generate a weighted voting system from the weighted average of two others, and that if the two parent games are in the same superregion, then so is the child.

After applying theorem 3 and setting $a=b$, the following is derived - if two points are in the same region, then any point on the line connecting those two points is also in the region. Therefore, given any two points in a region, all points on the line segment connecting those two points are also in the region. Thus, all regions must be convex. Alternatively, one may have noted that regions must be convex because they are the solution set to a system of linear inequalities.

The regions in the simplex are bounded by hyperplanes representing each possible coalition's weight being equal to the quota and by the facets of the simplex, which represent an individual's weight being equal to 0 . Thus, there is always a finite number of regions whose shapes are dynamically related with the quota. As the quota changes, the shape of the regions change, since some points in the simplex originally belonging to one superregion will now belong to a different superregion. It is interesting to see how the shapes of regions change as the quota changes.

It is noticeable that some superregions exist for only a subinterval of the complete quota interval $\left(\frac{1}{2}, 1\right]$. This phenomenon is easily explained by the system of inequalities that a region must satisfy. At certain values of the quota, the solution set to the system of inequalities might be empty - at these values for the quota, the superregion is said not to exist. In the geometric representation, it can be observed that when two hyperplanes that the region must lie between slide past one another, the region ceases to exist.

All superregions exist at some point in the quota interval $\left(\frac{1}{2}, 1\right]$, but not all superregions exist during the entirety of that interval. In the complete analysis for four-player weighted voting systems, it was important to notice which 
superregions were bounded by a quota interval narrower than $\left(\frac{1}{2}, 1\right]$. In four-player weighted voting systems, regions can have between 4 and 10 faces, each face representing a plane. It is curious how some regions' shapes changed along with the quota. Though a region is always bounded simultaneously by all of the inequalities that characterize it, some of the half spaces defined within the simplex by the inequalities may be strictly contained in others at certain values of the quota, so the number of planes that actually bound the region in the geometric representation may be less than the number of inequalities that characterize it.

This paper refers to the values of the quota for which a superregion exists as the "quota range" of that superregion. The "Quota Sandwich Theorem" may also be used to mitigate the problem of finding the quota range. It is stated and proved below.

Theorem 4 (Quota Sandwich Theorem) If a superregion exists when $q=a$ and when $q=b$, then the region exists in the interval $q \in[a, b]$.

Proof. After applying theorem 3, it can be seen that weighted voting systems with all values of quota in the closed interval $[a, b]$ can be constructed.

The quota sandwich theorem indicates that a superregion's quota range will not have any discontinuities, and suggests that the quota may be added to the geometric representation as an additional dimension, allowing the superregions to be represented. Indeed, if the quota were added as an $n$-th dimension, the complete geometric representation for $n$-player weighted voting systems will have been created. It is a prism created by "stacking" all of the geometric representations for $n$-player weighted voting systems at all feasible values of the quota. The complete geometric representation for $n$-player weighted voting systems is truly complete since it includes all possible weighted voting systems for $n$-players.

\subsection{Applying the Geometric Representation and Regions}

The geometry of weighted voting systems can be extensively analyzed using regions. The most obvious advantage for using regions is to take advantage of their completeness in fixed-quota circumstances. Since regions partition the simplex, every single point in the simplex belongs to a certain region. By looking at a simplex where the regions have been marked, one can ascertain information regarding weighted voting systems at the chosen value of the quota. It becomes possible to determine the answer to questions such as "given a fixed value for the quota and a random weight vector, what is the probability of a certain power distribution occurring?"

The use of regions and superregions in the geometric representation of weighted voting systems is also very powerful in dispelling paradoxes of voting power, as demonstrated in Jones (2009). When the weight vector of a weighted voting system, its quota, or both change, no alteration will be made to the original weighted voting system unless it has moved to another superregion. When the weight vector is modified, the chosen point will move, and if it crosses a hyperplane, it will arrive in a new region, with new power distributions. Altering the quota produces a similar effect, since the location of the hyperplanes will become altered. Regions greatly simplify the analysis of weighted voting systems because they represent a fixed number of states. A weighted voting system can be and must be in exactly one region, yet the number of regions is finite, fixing the number of possible states.

Tolle (2003) asks "for weighted voting systems of size $n$, is there a formula in terms of $n$ for the number of feasible power distributions?" and "with a complete enumeration of the power distributions feasible for weighted voting systems of size $n$, can one efficiently generate a complete list of feasible power distributions for size $n+1$ weighted voting systems?" Several papers have tried to answer Tolle's questions, including Brown et al. (2009), Cuttler et al. (2005), Elkind et al. (2005), and Keijzer et al. (2010), though often, their definition of "feasible power distribution" was different from Tolle's one.

These papers either tried to establish various properties about the number of feasible power distributions for $n$ player weighted voting systems or presented an algorithm which would enumerate the complete set of feasible sets of minimum winning coalitions. The geometric representation combined with the concept of superregions, however, might be able to simplify this analysis. Indeed, the geometric representations for $n$-player weighted voting systems can be used to generate $(n+1)$-player weighted voting systems since the faces of a $(n)$-simplex, representing $(n+1)$-player weighted voting systems, are $(n-1)$-simplexes, representing $n$-player weighted voting systems. Instead of algebraic or set manipulation, it becomes necessary only to calculate the number of distinct superregions bounded by hyperplanes in the prism representing the complete geometric representation for $n$-player weighted voting systems. If such an enumeration were found, the "inverse problem" of discovering the weighted voting system returning a power distribution closest to the desired one would also be solved. 


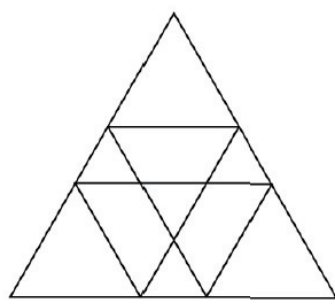

(a) $q<\frac{2}{3}$

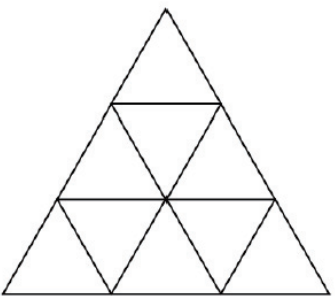

(b) $q=\frac{2}{3}$

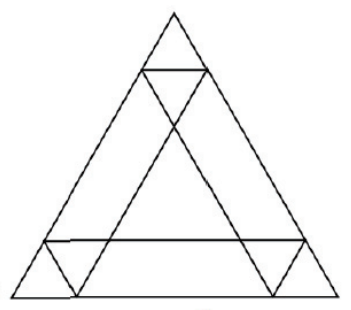

(c) $q>\frac{2}{3}$

Figure 1. The geometric representation for 3-player weighted voting systems

\section{Three Player Weighted Voting Systems}

\subsection{Overview}

Before proceeding to 4-Player Weighted Voting Systems, 3-Player Weighted Voting Systems must be analyzed. Much work was done on 3-player weighted voting systems by Kirstein (2009) and Jones (2009). The latter used a geometric analysis to reveal that 11 different superregions exist in total, a maximum of 10 of which could exist at any one given time.

The geometric representation of 3-player weighted voting systems at a specific quota are 2-simplexes, or equilateral triangles. There are two planes parallel to each side of the triangle, making six in total; these planes partition the simplex into the regions. As the quota changes, the regions' shapes also change (see Figure 1).

Table 1. The five unique region types for 3-player weighted voting systems

\begin{tabular}{cccc}
\hline Region Type \# & Minimum Winning Coalitions & Banzhaf Power Distribution & Quota Range \\
\hline 1 & {$[A]$} & {$[1,0,0]$} & $\left(\frac{1}{2}-1\right]$ \\
2 & {$[A B]$} & {$\left[\frac{1}{2}, \frac{1}{2}, 0\right]$} & $\left(\frac{1}{2}-1\right]$ \\
3 & {$[A B][A C]$} & {$\left[\frac{3}{5}, \frac{1}{5}, \frac{1}{5}\right]$} & $\left(\frac{1}{2}-1\right)$ \\
4 & {$[A B][A C][B C]$} & {$\left[\frac{1}{3}, \frac{1}{3}, \frac{1}{3}\right]$} & $\left(\frac{1}{2}-\frac{2}{3}\right]$ \\
5 & {$[A B C]$} & {$\left[\frac{1}{3}, \frac{1}{3}, \frac{1}{3}\right]$} & $\left(\frac{2}{3}-1\right]$ \\
\hline
\end{tabular}

Table 1 lists not regions, but region types. Two regions (or superregions) are said to be of the same type if the set of minimum winning coalitions of one can be changed into the set of minimum winning coalitions of the other by permuting the players. A region type is represented by the region of that type that satisfies the canonic rule. The canonic rule states that a player cannot have a weight greater than a player who comes before him or her in the alphabet. Hence, $A \geq B \geq C \geq \ldots$. Regions and superregions of the same type exhibit many of the same properties, like quota range.

Although there are only 5 region types for 3-player weighted voting systems, 11 superregions exist in total since there are 3 superregions of region types 1,2, and 3 and 1 superregion of region types 4 and 5 . A maximum of 10 superregions may exist at once because the regions of type 4 and those of type 5 exclude one another.

Regions of type 1, type 2, and type 3 exist at all values of the quota. More interesting is the relationship between the regions of type 4 and type 5, whose quota domains complement one another's: when the quota is infinitesimally greater than $\frac{1}{2}$, the region of type 4 begins as an upside-down triangle. When the quota is exactly $\frac{2}{3}$, that has shrunk to a point at the center of the simplex. As the quota grows larger than $\frac{2}{3}$, the region of type 5 replaces the region of type 4, and it takes the shape of a triangle that grows to eventually fill the entire simplex when the quota is 1 .

\subsection{The Complete Geometric Representation}

The quota, of course, has just as much influence on the geometric representation of weighted voting systems as the weight vector does, perhaps even more. Changing the weight vector amounts to changing the location of a point, which could place it in a different region, but changing the quota will change the shape and number of regions. Therefore, as stated previously, a better and more complete geometric representation could be made by including the quota as an additional dimension. The prism created by adding an additional dimension to the geometric 
representation of all weighted voting systems at a particular quota is powerful because of its completeness geometric analysis using the prism may prove superior to algebraic manipulation.

Jones (2009) uses both the motion of planes representing change in the quota and the motion of the point representing the weight vector to explain paradoxes of weighted voting, but if the prism were used, then there would be no need for the motion of planes since the complete geometric representation displays superregions instead of regions.

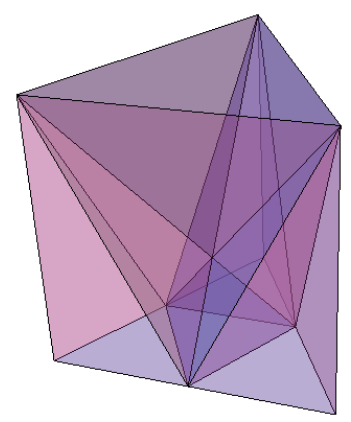

Figure 2. The complete geometric representation for 3-player weighted voting systems

Figure 2 displays the 11 superregions in the complete geometric representation for 3-player weighted voting systems. Each cross-section of this prism parallel to the bases is a simplex, which represents the weighted voting system with 3 players and the quota represented by the height of the cross-section. The top of the prism represents a quota of 1 , and the bottom represents a quota of $\frac{1}{2}$.

Every single weighted voting system with 3 players can be represented as a point in the above prism; thus, it is the complete geometric representation of 3 player weighted voting systems.

\subsection{A Brief Example}

Since the weight vector can change continuously while the power distribution can only take on discrete values, one may ask: "How large a change in the weight vector is needed to produce a change in the power distribution?" The Geometric Representation is great to answer this question. In corporate boards where shareholders engage in weighted voting by share size, ambitious shareholders who wish to increase their influence over company policy might ask this question.

Consider a company with three principal shareholders. Call them $A, B$, and $C$. The company implements weighted voting where each shareholder casts a number of votes proportional to the number of shares of stock they own. The company has one hundred total shares of stocks and implements a weighted voting system with the quota fixed at $q=60$ shares where each player has a number of votes equal to the number of shares of stock they own. Players $A$ and $C$ possess 30 shares each while player $B$ possesses 40 shares. One day, player $B$ comes to player $A$ and offers to sell some or all of his shares. Player $A$ is interested in increasing his influence over the company's decisions but wishes to purchase no more shares than are necessary. What are his choices?

With the help of the geometric representation, this analysis becomes simple. Examine Figure 3.

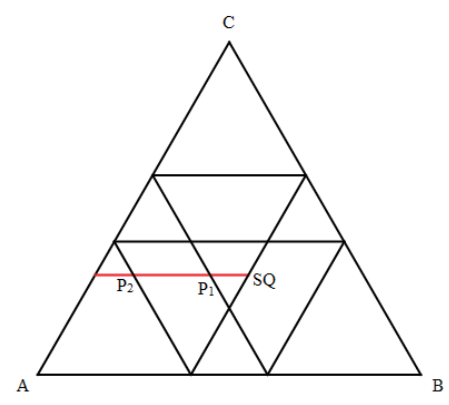

Figure 3. The geometric representation for the shareholder problem 
The geometric representation in Figure 3 has each player's "ideal" point labeled. At these points, the corresponding shareholder holds all of the shares. At the status quo (SQ), the normalized weighted voting system is $[0.6 ; 0.3,0.4,0.3]$. One can easily check that this weighted voting system falls in region type 4 , with the power distribution $\left[\frac{1}{3}, \frac{1}{3}, \frac{1}{3}\right]$.

The red line on the figure shows all points on the simplex that $A$ can reach through purchasing shares from $B$. From the geometric representation, it is clear that $A$ can increase his power only if he crosses a hyperplane dividing two regions. He can do just that by moving to point $P_{1}$ or point $P_{2}$. At the former, he purchases 11 shares and gains $\frac{4}{15}$ power. At the latter, he purchases 30 shares and gains $\frac{2}{3}$ power. Thus, it can be summarized that $A$ has three optimal choices:

$$
\begin{aligned}
& \text { SQ: }[0.6 ; 0.3,0.4,0.3] \rightarrow \text { Region }[A B][A C][B C] \rightarrow\left[\frac{1}{3}, \frac{1}{3}, \frac{1}{3}\right] ; \\
& P_{1}:[0.6 ; 0.41,0.29,0.3] \rightarrow \text { Region }[A B][A C] \rightarrow\left[\frac{3}{5}, \frac{1}{5}, \frac{1}{5}\right] ; \\
& P_{2}:[0.6 ; 0.60,0.1,0.3] \rightarrow \text { Region }[A] \rightarrow[1,0,0] .
\end{aligned}
$$

Depending on how much power $A$ desires and how much he values an increase in power, $A$ will choose between these three situations, since these situations are the only rational outcomes.

\section{Four-Player Weighted Voting Systems}

\subsection{The Planes}

With four players, the 3-simplex is used, which is a tetrahedron with unit height. A total of 18 planes bound the regions. These planes are represented in Table 2. Note that simplex coordinates, $(A, B, C, D)$, are being used, which label each point with the distance from that point to each face.

Table 2. Types of planes bounding regions for 4-player weighted voting systems

\begin{tabular}{lr}
\hline Plane Type & Equation of Planes \\
\hline Four Faces of Tetrahedron & $A=0 ; B=0 ; C=0 ; D=0$ \\
1-P Coalitions & $A=q ; B=q ; C=q ; D=q$ \\
2-P Coalitions & $A B=q ; A C=q ; A D=q ; B C=q ; B D=q ; C D=q$ \\
3-P Coalitions & $A B C=q ; A B D=q ; A C D=q ; B C D=q$ \\
\hline
\end{tabular}

Of these planes, 14 are planes representing a coalition's weight equaling the quota. These planes are called quotaplanes and are detailed in Figure 4, Figure 5, and Figure 6.

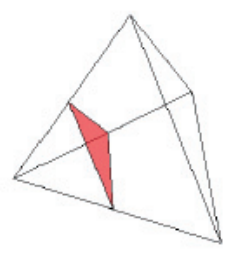

(a) $A=q$

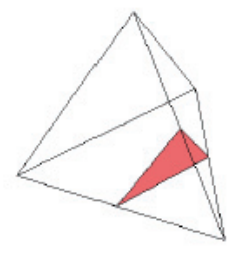

(b) $B=q$

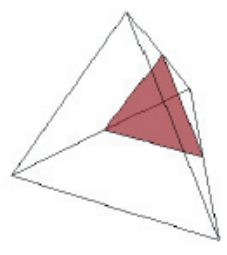

(c) $C=q$

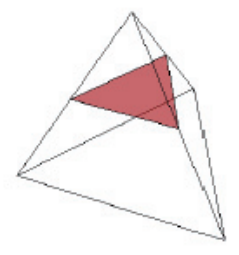

(d) $D=q$

Figure 4. The 1-player quota-planes 


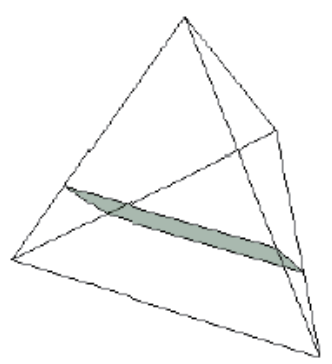

(a) $A B=q$

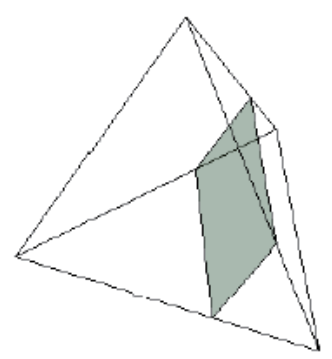

(d) $B C=q$

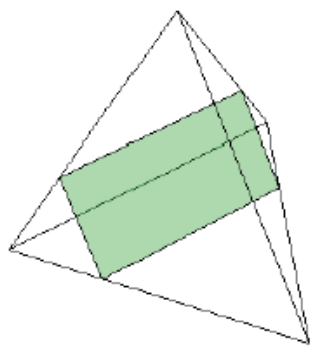

(b) $A C=q$

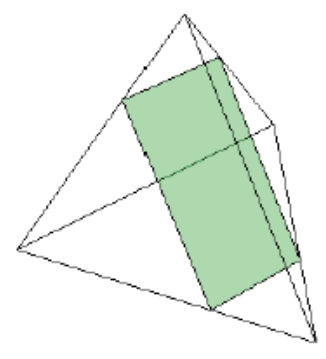

(e) $B D=q$

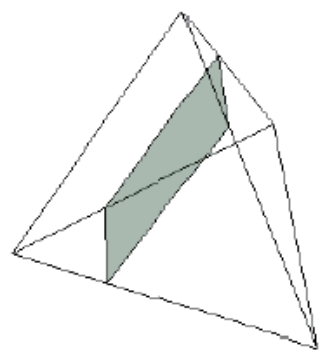

(c) $A D=q$

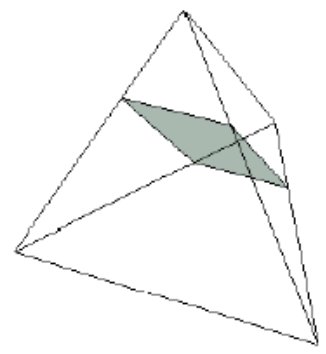

(f) $C D=q$

Figure 5. The 2-player quota-planes

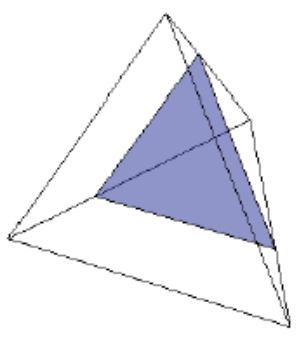

(a) $A B C=q$

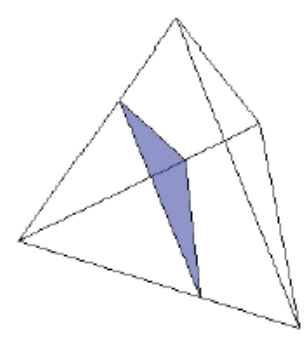

(b) $A B D=q$

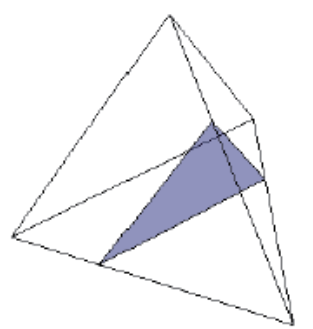

(c) $A C D=q$

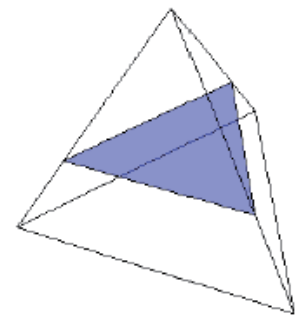

(d) $B C D=q$

Figure 6. The 3-player quota-planes

An interesting observation is that the planes corresponding to the 1-P coalitions are parallel to the planes corresponding to the 3-P coalitions. This fact is due to the universal equation $\mathrm{ABCD}=1$, which must always be satisfied. Thus, the 3-P coalitions could be represented as $A=1-q ; B=1-q ; C=1-q ; D=1-q$, which are equations producing planes parallel to those of the 1-P coalitions. These planes, in turn, are also parallel to the faces of the tetrahedron. The planes representing 2-P coalitions also form parallel pairs. The plane representing each coalition's weight equaling the value of the quota is parallel to the plane corresponding to the coalition's complement's weight equaling the quota.

\subsection{The 3-Simplex}

In Figure 7, there are nine 3-simplexes which are the geometric representation for 4-player weighted voting systems at various values of the quota. Although a more complete representation could be achieved by adding a fourth dimension to the simplex, such a figure would be difficult to display. The planes colored red are $A=q, B=q$, $C=q$, and $D=q$. The green planes are $A B=q, A C=q, A D=q, B C=q, B D=q$, and $C D=q$. The blue planes are $A B C=q, A B D=q, A C D=q$, and $B C D=q$. This coloring scheme will be maintained throughout the paper.

In four-player games, there are 80 different legal sets of minimum winning coalitions, creating 80 theoretically possible superregions in total. Though all superregions exist at some values of the quota, not all of these superregions can exist as regions at the same time. The maximum number of regions which can coexist under a fixed 
quota is 65 . When $q \in\left(\frac{1}{2}, \frac{2}{3}\right)$, the number of regions is 65 . When $q \in\left[\frac{2}{3}, 1\right)$, the number of regions is 53 . When $q=1$, the number of regions is 15 . The 80 total possible regions fall into 14 region types. These 14 region types are outlined in Table 3 and Table 4.

Table 3. Region Types in 4-Player weighted voting systems

\begin{tabular}{cccc}
\hline Region Type \# & Minimum Winning Coalitions & Banzhaf Power Distribution & Quota Range \\
\hline 1 & {$[A]$} & {$[1,0,0,0]$} & $\left(\frac{1}{2}, 1\right]$ \\
2 & {$[A B]$} & {$\left[\frac{1}{2}, \frac{1}{2}, 0,0\right]$} & $\left(\frac{1}{2}, 1\right]$ \\
3 & {$[A B][A C]$} & {$\left[\frac{3}{5}, \frac{1}{5}, \frac{1}{5}, 0\right]$} & $\left(\frac{1}{2}, 1\right)$ \\
4 & {$[A B][A C][A D]$} & {$\left[\frac{7}{10}, \frac{1}{10}, \frac{1}{10}, \frac{1}{10}\right]$} & $\left(\frac{1}{2}, 1\right)$ \\
5 & {$[A B][A C D]$} & {$\left[\frac{1}{2}, \frac{3}{10}, \frac{1}{10}, \frac{1}{10}\right]$} & $\left(\frac{1}{2}, 1\right)$ \\
6 & {$[A B][A C][B C]$} & {$\left[\frac{1}{3}, \frac{1}{3}, \frac{1}{3}, 0\right]$} & $\left(\frac{1}{2}, \frac{2}{3}\right]$ \\
7 & {$[A B][A C][B C D]$} & {$\left[\frac{5}{12}, \frac{1}{4}, \frac{1}{4}, \frac{1}{12}\right]$} & $\left(\frac{1}{2}, \frac{2}{3}\right)$ \\
8 & {$[A B][A C D][B C D]$} & {$\left[\frac{1}{3}, \frac{1}{3}, \frac{1}{6}, \frac{1}{6}\right]$} & $\left(\frac{1}{2}, \frac{2}{3}\right]$ \\
9 & {$[A B C]$} & {$\left[\frac{1}{3}, \frac{1}{3}, \frac{1}{3}, 0\right]$} & $\left(\frac{2}{3}, 1\right]$ \\
10 & {$[A B C][A B D]$} & {$\left[\frac{3}{8}, \frac{3}{8}, \frac{1}{8}, \frac{1}{8}\right]$} & $\left(\frac{2}{3}, 1\right)$ \\
11 & {$[A B][A C][A D][B C D]$} & {$\left[\frac{1}{2}, \frac{1}{6}, \frac{1}{6}, \frac{1}{6}\right]$} & $\left(\frac{1}{2}, \frac{3}{5}\right]$ \\
12 & {$[A B C][A B D][A C D]$} & {$\left[\frac{2}{5}, \frac{1}{5}, \frac{1}{5}, \frac{1}{5}\right]$} & $\left(\frac{3}{5}, 1\right)$ \\
13 & {$[A B C][A B D][A C D][B C D]$} & {$\left[\frac{1}{4}, \frac{1}{4}, \frac{1}{4}, \frac{1}{4}\right]$} & $\left(\frac{1}{2}, \frac{3}{4}\right]$ \\
14 & {$[A B C D]$} & {$\left[\frac{1}{4}, \frac{1}{4}, \frac{1}{4}, \frac{1}{4}\right]$} & $\left(\frac{3}{4}, 1\right]$ \\
\hline
\end{tabular}

Table 4. Quota Ranges in 4-Player weighted voting systems

\begin{tabular}{|c|c|c|c|c|}
\hline Region \# & \multicolumn{4}{|c|}{ Quota Range } \\
\hline & $\frac{1}{2}-\frac{3}{5}$ & $\frac{3}{5}-\frac{2}{3}$ & $\frac{2}{3}-\frac{3}{4}$ & $\frac{3}{4}-1$ \\
\hline 1 & \multicolumn{4}{|l|}{$[\mathrm{A}]$} \\
\hline 2 & \multicolumn{4}{|l|}{$[\mathrm{AB}]$} \\
\hline 3 & \multicolumn{4}{|l|}{$[\mathrm{AB}][\mathrm{AC}]$} \\
\hline 4 & \multicolumn{4}{|c|}{$[\mathrm{AB}][\mathrm{AC}][\mathrm{AD}]$} \\
\hline 5 & \multicolumn{4}{|l|}{$[\mathrm{AB}][\mathrm{ACD}]$} \\
\hline 6 & \multicolumn{4}{|c|}{$[\mathrm{AB}][\mathrm{AC}][\mathrm{BC}]$} \\
\hline 7 & \multicolumn{4}{|c|}{$[\mathrm{AB}][\mathrm{AC}][\mathrm{BCD}]$} \\
\hline 8 & \multicolumn{4}{|c|}{$[\mathrm{AB}][\mathrm{ACD}][\mathrm{BCD}]$} \\
\hline 9 & \multicolumn{4}{|c|}{$[\mathrm{ABC}]$} \\
\hline 10 & \multicolumn{4}{|c|}{$[\mathrm{ABC}][\mathrm{ABD}]$} \\
\hline 11 & \multicolumn{4}{|l|}{$\begin{array}{l}{[\mathrm{AB}][\mathrm{AC}]} \\
{[\mathrm{AD}][\mathrm{BCD}]}\end{array}$} \\
\hline 12 & & \multicolumn{3}{|c|}{$[\mathrm{ABC}][\mathrm{ABD}][\mathrm{ACD}]$} \\
\hline 13 & \multicolumn{4}{|c|}{$[\mathrm{ABC}][\mathrm{ABD}][\mathrm{ACD}][\mathrm{BCD}]$} \\
\hline 14 & & & & {$[\mathrm{ABCD}]$} \\
\hline
\end{tabular}




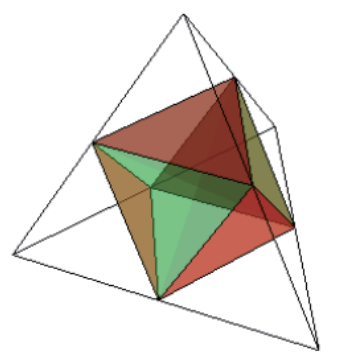

(a) $q=\frac{1}{2}+\epsilon$

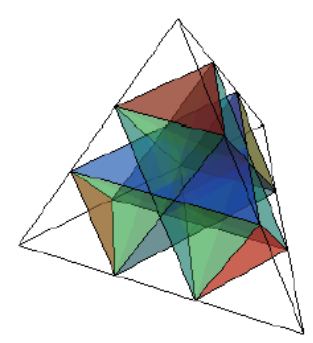

(d) $q=\frac{16}{25}$

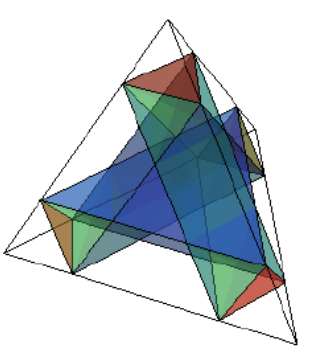

(g) $q=\frac{3}{4}$

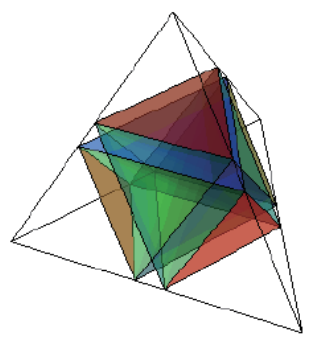

(b) $q=\frac{11}{20}$

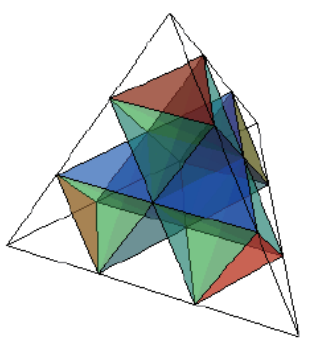

(e) $q=\frac{2}{3}$

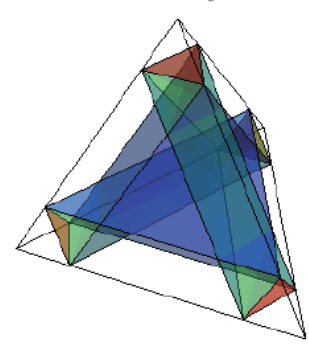

(h) $q=\frac{4}{5}$

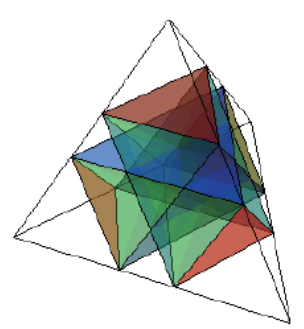

(c) $q=\frac{3}{5}$

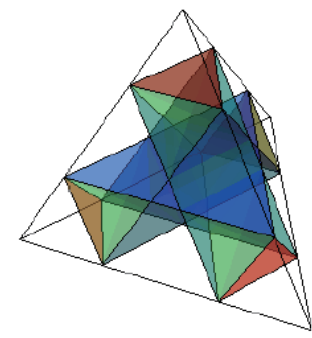

(f) $q=\frac{7}{10}$

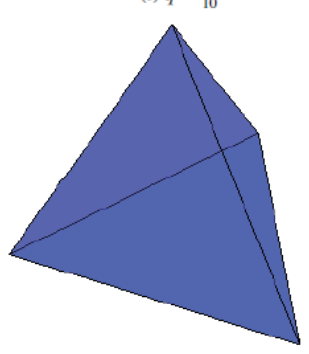

(i) $q=1$

Figure 7. The geometric representation for 4-player weighted voting systems

\subsection{Spotlight on Region Type 13}

Many superregions exist for most of the full quota range, but during their existence as regions, their shape constantly changes. As the quota changes, the position of the quota-planes move, but also, a region may suddenly become bounded by a different set of planes. The values of the quota at which a region can become bounded by different planes are the same values at which regions can appear or disappear. Regions belonging to some superregions have only one set of planes bounding them during their quota ranges, but some regions have two or three sets of planes that can bound them at various quota ranges.

The single superregion of region type 13 is one of the most interest superregions. It represents the superregion with majority rule and hence is symmetric. Nevertheless, the region actually changes shapes twice, giving it three sets of planes that bound it. The shapes depicted in Figure 8 are representative of the shapes this region could take.

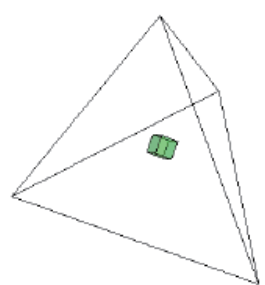

(a) $q=0.55$

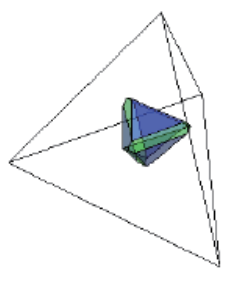

(b) $q=0.65$

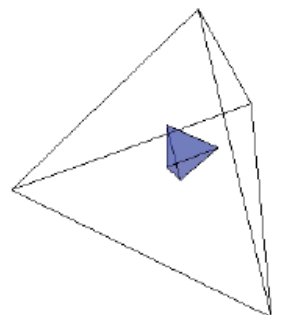

(c) $q=0.70$

Figure 8. Region type 13 
When the quota is between $q=\frac{1}{2}$ and $q=\frac{3}{5}$, this superregion is bounded by the 2-player quota-planes only. When the quota is greater than $q=\frac{3}{5}$ but not more than $q=\frac{2}{3}$, the most interesting shape results. The superregion is bounded by 10 planes, including all of the 2-player quota-planes and all of the 3-player quota-planes. Four corners of the cube that form a tetrahedron are cut by the 3-player quota-planes, creating a shape having four triangular faces and six hexagonal faces. When the quota exceeds $q=\frac{2}{3}$, the shape simplifies to a tetrahedron, inverted with respect to the simplex. It shrinks to a single point when the quota is $q=\frac{3}{4}$ and then disappears when the quota exceeds that value.

Information about the other 13 region types can be found in the appendixes. Appendix A will provide details about all 14 region types. Images can be found in Appendix B.

\subsection{Another Brief Example}

The geometric representation for four-player weighted voting systems can be applied to solve problems in the same way as the geometric representation for three-player weighted voting systems. Again, one question that the geometric representation can answer is "how much of a change in a weight vector is required to produce a change in the power distribution?"

Take the example of the corporation from subsection 5.3 again and assume that player $A$ chooses to stay at the status quo. One year later, an individual wishes to buy shares and become another shareholder (so that there will be four shareholders how). Player $B$ sells 20 shares to the fourth individual, who will be called player $D$. Player $A$ then offers to sell additional shares to player $D$. Now, player $D$ wishes to increase his influence over the company but purchase the least number of shares needed to do so. What are his options?

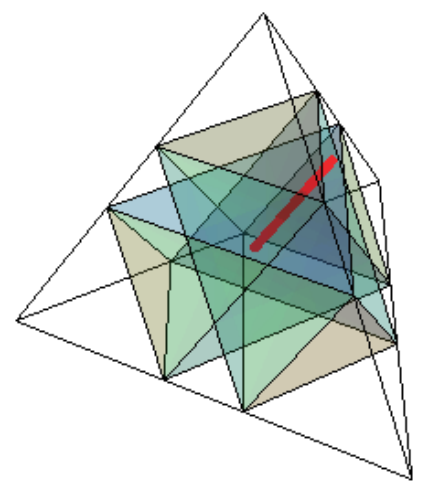

Figure 9. The geometric representation for the new shareholder's problem

The red line in Figure 9 notes all of the points in the simplex that player $D$ can attain. This line only intersects 5 different regions, listed below and detailed in Figure 10:

$$
\begin{aligned}
& \text { SQ: }[0.6 ; 0.3,0.2,0.3,0.2] \rightarrow \text { Region }[A C][A B D][B C D] \rightarrow\left[\frac{1}{3}, \frac{1}{6}, \frac{1}{3}, \frac{1}{6}\right] ; \\
& P_{1}:[0.6 ; 0.29,0.2,0.3,0.21] \rightarrow \text { Region }[A B C][A B D][A C D][B C D] \rightarrow\left[\frac{1}{4}, \frac{1}{4}, \frac{1}{4}, \frac{1}{4}\right] ; \\
& P_{2}:[0.6 ; 0.2,0.2,0.3,0.3] \rightarrow \text { Region }[C D][A B C][A B D] \rightarrow\left[\frac{1}{6}, \frac{1}{6}, \frac{1}{3}, \frac{1}{3}\right] ; \\
& P_{3}:[0.6 ; 0.1,0.2,0.3,0.4] \rightarrow \text { Region }[B D][C D][A B C] \rightarrow\left[\frac{1}{12}, \frac{1}{4}, \frac{1}{4}, \frac{5}{12}\right] ; \\
& P_{4}:[0.6 ; 0.09,0.2,0.3,0.41] \rightarrow \text { Region }[B D][C D] \rightarrow\left[0, \frac{1}{5}, \frac{1}{5}, \frac{3}{5}\right] .
\end{aligned}
$$

In summary, player $D$ may purchase 1 share to increase his power by $\frac{1}{12}, 10$ shares to increase his power by $\frac{1}{6}, 20$ shares to increase his power by $\frac{1}{4}$, or 21 shares to increase his power by $\frac{13}{30}$. 


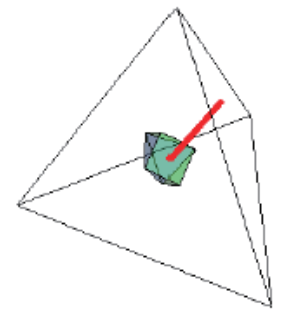

(a) SQ

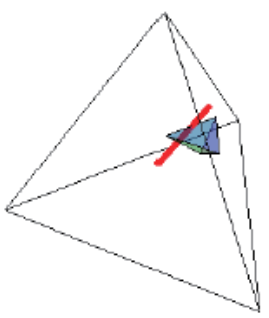

(d) P3

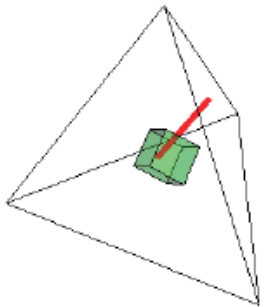

(b) P1

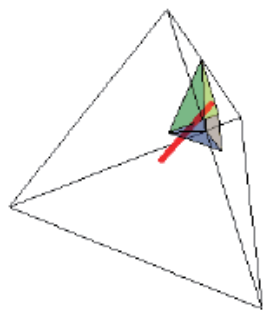

(e) P4

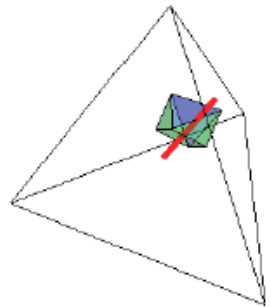

(c) $\mathrm{P} 2$

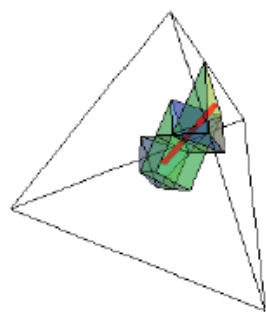

(f) All Solutions

Figure 10. The regions that are in the solution set to the new shareholder's problem

\section{Conclusion}

The analysis of weighted voting systems is a very broad topic. The use of the algebraic representation, while succinct, is sometimes inadequate and leaves many of the more intricate properties of weighted voting systems obscured - there is no easy method to see the boundaries of a weighted voting system or to assess the finiteness of the number of distinct weighted voting systems. The geometric representation, representing each weighted voting system as a point, assigns to each weighted voting system a clearly bounded region within a simplex and a superregion with characteristic properties, allowing the analysis of other interesting properties of weighted voting systems, such as their shape. The geometric representation of weighted voting systems has been used for the analysis of four-player weighted voting systems. The use of hyperplanes to limit these regions allows any changes in the quota and weights to be clearly shown by the motion of points and hyperplanes. An even better but more difficult to display representation involves making the quota an additional dimension - changes in quota would then be represented only by the motion of a point. The completeness of the geometric representation makes it a very useful alternative to the standard algebraic representation.

\section{References}

Banzhaf, J. (1965). Weighted Voting Doesn't Work: A Mathematical Analysis. Rutgers Law Review, 19(2), 317343.

Bishnu, M., \& Roy, S. (2009). Hierarchy of Players in Swap Robust Voting Games and Minimal Winning Coalitions. Working paper. Ames, Iowa: Iowa State University Department of Economics.

Brown, E., Donovan, C., \& Noneman, C. (2009). Weighted Voting Systems. Carleton College Math Comps.

Cuttler, A. B., Guire, A. L. De, \& Rowell, S. K. (2005). Using Sets of Winning Coalitions to Generate Feasible Banzhaf Power Distributions. Proc. of 2005 Undergraduate Mathematics Day at the University of Dayton.

Elkind, E., Goldberg, L. A., Goldberg, P. W., \& Wooldridge, M. (2009). On the Computational Complexity of Weighted Voting Games. Annals of Mathematics, 56(2), 109-31. http://dx.doi.org/10.1007/s10472-009-9162-5

Jones, M. A. (2009). The Geometry Behind Paradoxes of Voting Power. Mathematics Magazine, 82(2), 103-116. http://dx.doi.org/10.4169/193009809X468913

Keijzer, B. D., Klos, T., \& Zhang, Y. Q. (2010). Enumeration and Exact Design of Weighted Voting Games. Proc. of 9th International Conference on Autonomous Agents and Multiagent Systems, 1, 391-398. 
Kirsch, W., \& Langner, J. (2010). Power Indices and Minimal Winning Coalitions. Social Choice and Welfare, 34(1), 33-46. http://dx.doi.org/10.1007/s00355-009-0387-3

Kirstein, R. (2009). Volkswagen vs. Porsche: A Power-Index Analysis. International Journal of Corporate Governance, 2(1), 1-20. http://dx.doi.org/10.2139/ssrn.1420742

Laruelle, A., \& Valenciano, F. (2005). A Critical Reappraisal of Some Voting Power Paradoxes. Public Choice, 125(1-2), 17-41. http://dx.doi.org/10.1007/s11127-005-3408-5

Tolle, J. (2003). Power Distribution in Four-Player Weighted Voting Systems. Mathematics Magazine, 76(1), 33-39. http://dx.doi.org/10.2307/3219130

\section{Appendix A: Descriptions of the Region Types in Four-Player Weighted Voting Systems}

a. Region Type 1

MWCs: $[A]$

Power: $[1,0,0,0]$

This region is always shaped like a tetrahedron and exists for the full quota range.

b. Region Type 2

MWCs: $[A B]$

Power: $\left[\frac{1}{2}, \frac{1}{2}, 0,0\right]$

This region's shape is pyramidal when the quota satisfies $\frac{1}{2}<q \leq \frac{2}{3}$. When the quota exceeds $\frac{2}{3}$, however, the region becomes a wedge-like shape.

c. Region Type 3

MWCs: $[A B][A C]$

Power: $\left[\frac{3}{5}, \frac{1}{5}, \frac{1}{5}, 0\right]$

This region's shape is wedge-like when the quota satisfies $\frac{1}{2}<q<\frac{3}{5}$. When the quota satisfies $\frac{3}{5}<q<\frac{2}{3}$, the region's shape is also wedge-like, but the orientation is different. When $q=\frac{3}{5}$ or $q \geq \frac{2}{3}$, the region's shape is pyramidal.

d. Region Type 4

MWCs: $[A B][A C][A D]$

Power: $\left[\frac{7}{10}, \frac{1}{10}, \frac{1}{10}, \frac{1}{10}\right]$

This region's shape is like the frustum of a triangular pyramid when the quota satisfies $\frac{1}{2}<q<\frac{3}{5}$. When the quota exceeds $\frac{3}{5}$, however, the region becomes a pyramidal shape.

e. Region Type 5

MWCs: $[A B][A C D]$

Power: $\left[\frac{1}{2}, \frac{3}{10}, \frac{1}{10}, \frac{1}{10}\right]$

This region's shape is pyramidal when the quota satisfies $\frac{1}{2}<q \leq \frac{3}{5}$. When the quota satisfies $\frac{3}{5}<q<\frac{2}{3}$, the region's shape is wedge-like. When $q \geq \frac{2}{3}$ the region's shape is once again pyramidal, but its orientation is different.

f. Region Type 6

MWCs: $[A B][A C][B C]$

Power: $\left[\frac{1}{3}, \frac{1}{3}, \frac{1}{3}, 0\right]$

This region's shape is pyramidal and it exists for the quota range $\frac{1}{2}<q \leq \frac{2}{3}$.

g. Region Type 7

MWCs: $[A B][A C][B C D]$ 
Power: $\left[\frac{5}{12}, \frac{1}{4}, \frac{1}{4}, \frac{1}{12}\right]$

This region takes a wedge-like shape when the quota satisfies $\frac{1}{2}<q<\frac{3}{5}$. When the quota exceeds $\frac{3}{5}$, however, the region becomes a pyramidal shape. This region disappears when the quota is any larger than $q=\frac{2}{3}$.

h. Region Type 8

MWCs: $[A B][A C D][B C D]$

Power: $\left[\frac{1}{3}, \frac{1}{3}, \frac{1}{6}, \frac{1}{6}\right]$

The shape of this region is very strange. When the quota satisfies $\frac{1}{2}<q<\frac{3}{5}$, the region has seven faces, five quadrilateral and two triangular. When the quota exceeds $\frac{3}{5}$, the region becomes a different strange shape with seven faces. It has one hexagonal face, two quadrilateral faces, and four triangular faces. This region shrinks to a point when $q=\frac{2}{3}$ and disappears when the quota is any larger.

i. Region Type 9

MWCs: $[A B C]$

Power: $\left[\frac{1}{3}, \frac{1}{3}, \frac{1}{3}, 0\right]$

This region does not exist when the quota is small. When the quota satisfies $\frac{2}{3}<q \leq \frac{3}{4}$, the region takes the form of a tetrahedron. When the quota exceeds $\frac{3}{4}$, however, the region suddenly becomes cut from above, changing its shape into that of the frustum of a pyramid. It continues to exist, even when $q=1$.

j. Region Type 10

MWCs: $[A B C][A B D]$

Power: $\left[\frac{3}{8}, \frac{3}{8}, \frac{1}{8}, \frac{1}{8}\right]$

This region has a wedge-like shape when the quota satisfies $\frac{2}{3}<q<\frac{3}{4}$. At $q=\frac{3}{4}$, the shape is briefly pyramidal, but when $\frac{3}{4}<q<1$, the shape is once again wedge-like, but with different orientation. This second orientation forms a right angle with the first orientation.

k. Region Type 11

MWCs: $[A B][A C][A D][B C D]$

Power: $\left[\frac{1}{2}, \frac{1}{6}, \frac{1}{6}, \frac{1}{6}\right]$

This region's shape is pyramidal and it exists for the quota range $\frac{1}{2}<q \leq \frac{3}{5}$.

l. Region Type 12

MWCs: $[A B C][A B D][A C D]$

Power: $\left[\frac{2}{5}, \frac{1}{5}, \frac{1}{5}, \frac{1}{5}\right]$

This region's shape begins pyramidal when the quota satisfies $\frac{3}{5}<q \leq \frac{2}{3}$. When the quota satisfies $\frac{2}{3}<q<\frac{3}{4}$, the region has seven faces. Its shape can be described as a pyramid glued to the frustum of a pyramid at their bases. When the quota satisfies $\frac{3}{4} \leq q<1$, the region becomes a double pyramid.

m. Region Type 13

MWCs: $[A B C][A B D][A C D][B C D]$

Power: $\left[\frac{1}{4}, \frac{1}{4}, \frac{1}{4}, \frac{1}{4}\right]$

This region only exists for the quota range $\frac{1}{2}<q \leq \frac{2}{3}$. When $\frac{1}{2}<q \leq \frac{3}{5}$, it is a cube. When $\frac{3}{5}<q<\frac{2}{3}$, it is a ten-faced shape with four triangular faces and six hexagonal faces. When $\frac{2}{3} \leq q<\frac{3}{4}$, the region is an inverted tetrahedron. The region is a single point when $q=\frac{3}{4}$ and disappears when the quota is any larger.

n. Region Type 14

MWCs: $[A B C D]$

Power: $\left[\frac{1}{4}, \frac{1}{4}, \frac{1}{4}, \frac{1}{4}\right]$

This region only exists for the quota range $\frac{3}{4}<q \leq 1$. It is always shaped like a tetrahedron and grows to fill the 
interior of the simplex when $q=1$.

Appendix B: Images of the Region Types in Four-Player Weighted Voting Systems

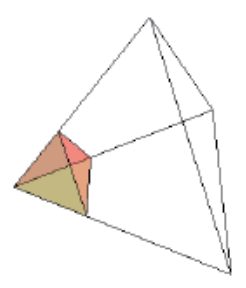

(a) $q=0.64$

Figure 11. Region type 1

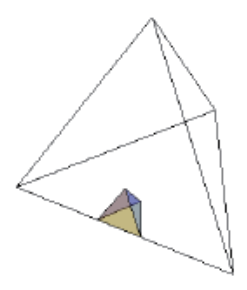

(a) $q=0.60$

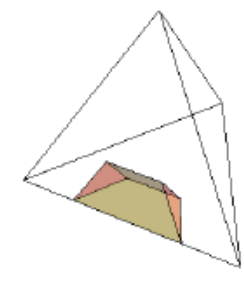

(b) $q=0.75$

Figure 12. Region type 2

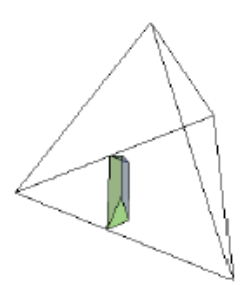

(a) $q=0.55$

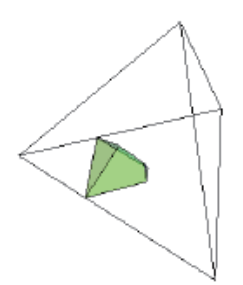

(b) $q=0.64$

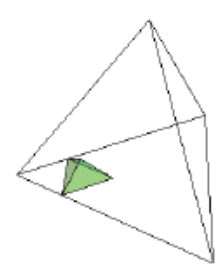

(c) $q=0.75$

Figure 13. Region type 3

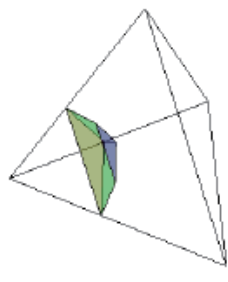

(a) $q=0.55$

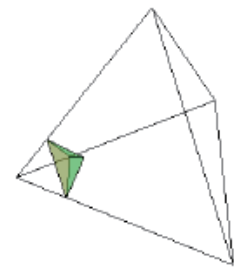

(b) $q=0.75$

Figure 14. Region type 4

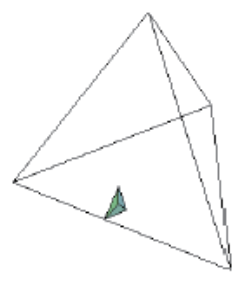

(a) $q=0.55$

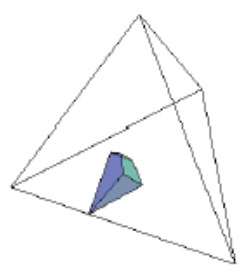

(b) $q=0.63$

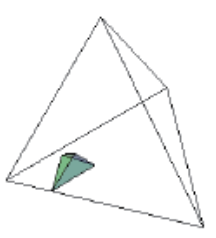

(c) $q=0.75$

Figure 15. Region type 5 


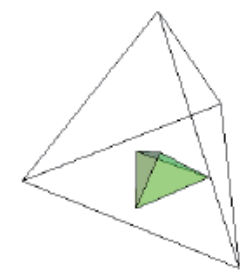

(a) $q=0.55$

Figre 16. Region type 6

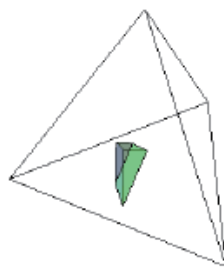

(a) $q=0.55$

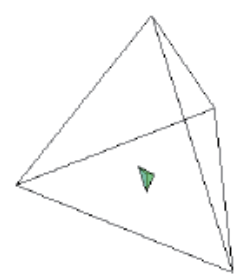

(b) $q=0.63$

Figure 17. Region type 7

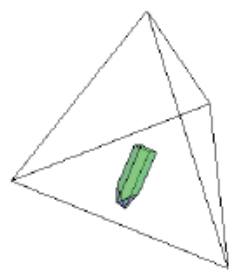

(a) $q=0.55$

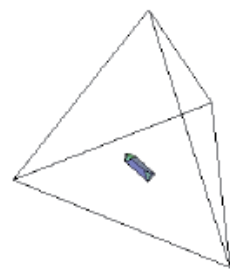

(b) $q=0.65$

Figure 18. Region type 8

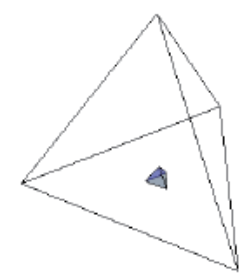

(a) $q=0.70$

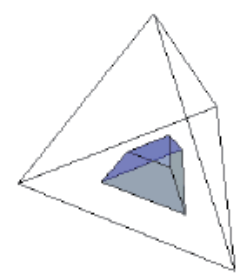

(b) $q=0.80$

Figure 19. Region type 9
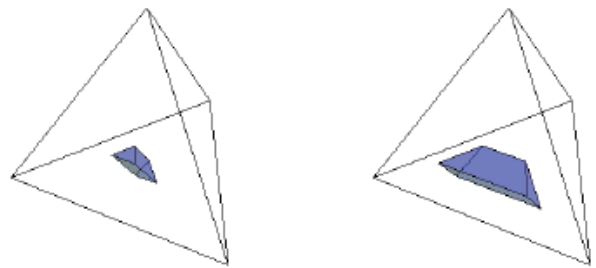

(b) $q=0.80$

Figure 20. Region type 10 


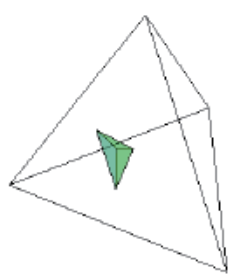

(a) $q=0.55$

Figure 21. Region type 11

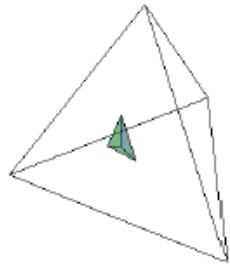

(a) $q=0.64$

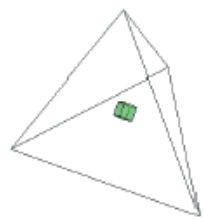

(a) $q=0.55$

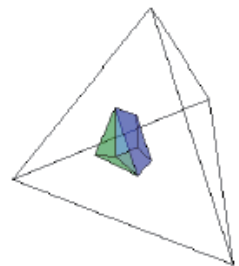

(b) $q=0.70$

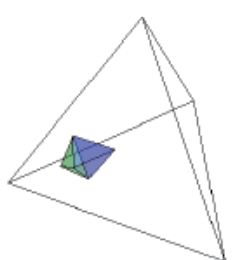

(c) $q=0.80$

Figure 22. Region type 12

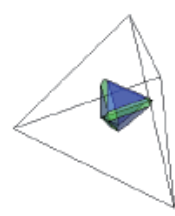

(b) $q=0.65$

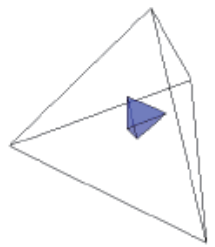

(c) $q=0.70$

Figure 23. Region type 13

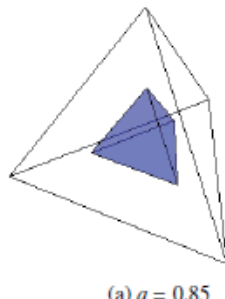

Figure 24. Region type 14 